\title{
Radiomics for prediction of survival in lower-grade gliomas-it's time to move beyond the crystal ball
}

\author{
Daniel Pinto dos Santos ${ }^{1}$. José Maria Garcia Santos ${ }^{2}$
}

Received: 13 November 2020 / Accepted: 4 December 2020 / Published online: 19 December 2020

(C) The Author(s) 2020

\section{Key Points}

- Radiomics might help predict survival of patients with lower-grade gliomas.

- Several different models using different radiomics features have been proposed with only little overlap in included features.

- Prospective trials and validation studies are needed to establish which models offer clinical benefit and which do not.

Lower-grade gliomas (LGG, WHO grades II and III) show a wide range of biologic aggressiveness. Accordingly, after the initial surgical resection, there may be a variable need for additional treatment. Decisions on further radiation and/or chemotherapy should be considered on an individual basis [1]. A recent survey in Germany demonstrated high heterogeneity between oncology centers in treatment decisions for LGG [2] and different guidelines have emphasized the idea that much more scientific evidence will be needed for treatment recommendations in the next future [3, 4].

Currently, two options can be considered for LGG when chemotherapy is needed after radiotherapy: PCV (procarbazine, CCNU [lomustine] and vincristine) or temozolomide. $\mathrm{PVC}$ is regarded as the more appropriate option in cases of grade II LGG needing treatment beyond surgery. In patients diagnosed with grade III gliomas, chemotherapy is usually contemplated after radiotherapy regardless of the extension of surgical resection or any other risk factor [1]; both PCV and temozolomide would benefit LLG outcomes [1, 3, 4]. Although there are currently no head-to-head comparison

This comment refers to the article available at https://oi.org/10.1007/ s00330-020-07581-3.

Daniel Pinto dos Santos

daniel.pinto-dos-santos@uk-koeln.de;

pintodossantos.daniel@gmail.com

1 Department of Radiology, University Hospital of Cologne, Kerpener Str. 62, 50937 Köln, Germany

2 Department of Radiology, Hospital General Universitario Morales Meseguer, Murcia, Spain between temozolomide and PCV, the former might be more appropriate and is less toxic [1].

While the current study by Wang et al [5] published in this number of European Radiology might not add much evidence as to what therapeutic strategy might be the most appropriate for each individual patient, they aimed at helping clinical decision-making by proposing a radiomics model to predict survival of patients with LGG. They tested their radiomics signature in LGG patients treated with temozolamide to conclude that it has the potential to discriminate patients who may benefit most from this chemotherapy regime.

Certainly, this is no particularly new topic but still their study adds to the idea that one day we might be able to provide individualized treatment decisions based on medical imaging. Recently, two other studies, one by Park et al [6] and one by Choi et al [7], also explored the idea to use radiomics in order to predict survival in patients with LGG. However, if we compare these three studies, one thing becomes very clear: three papers propose five radiomics models and no single radiomic feature is present in all five models. Over the last years, it has become clear, that radiomics research is facing a "reproducibility crisis" that has led to a significant "translation gap" of promising research results into clinical practice $[8,9]$.

Considering the abovementioned three studies, it is interesting to see that out of 176 features that were included in any of the five models, only five features ("original_glcm_idmn_t1c," "original_glcm_idmn_t2," "original_glcm_idn_t2," "original_firstorder_skewness_t1 c," and "original_glszm_smallareaemphasis_t1 c") were included in three of the five models. These features however, being calculated from contrast-enhanced $\mathrm{T} 1$ and $\mathrm{T} 2$ sequences respectively, might be subject to relevant test-retest variation [10], whereas many of the features included in the current 
study by Wang et al [5] were calculated from FLAIR sequences which have been shown to exhibit greater test-retest stability [10]. Even more interestingly, none of the features included in the final model of the study by Wang et al [5] was included in the other studies.

No doubt, radiomic analyses hold the promise to significantly impact patient management by allowing for noninvasive diagnosis and prognosis. However, given the large increase in publications pertaining to this topic and the abovementioned issues, it is difficult to ascertain which model from which publication could indeed be generalizable enough to impact clinical routine. Now might be the time, to move beyond using radiomics to look inside crystal balls and start evaluating already published radiomic models' predictions and trying to reproduce and understand their results. We have to be able to put promising research results to practical workand certainly the study by Wang et al [5] is an example of such promising results. This will certainly at some point involve putting such models to the test of prospective studies, in which their clinical value has to be proven in terms of impact on patient outcome. No easy feat of course, but only these next steps will help us move forward.

Funding Open Access funding enabled and organized by Projekt DEAL.

\section{Compliance with ethical standards}

Guarantor The scientific guarantor of this publication is PD Dr. Daniel Pinto dos Santos, University Hospital of Cologne (daniel.pinto-dossantos@uk-koeln.de).

Conflict of interest The authors of this manuscript declare no relationships with any companies, whose products or services may be related to the subject matter of the article.

Statistics and biometry No complex statistical methods were necessary for this paper.

Informed consent Not applicable.

Ethical approval Not applicable.

Methodology

- editorial

Open Access This article is licensed under a Creative Commons Attribution 4.0 International License, which permits use, sharing, adaptation, distribution and reproduction in any medium or format, as long as you give appropriate credit to the original author(s) and the source, provide a link to the Creative Commons licence, and indicate if changes were made. The images or other third party material in this article are included in the article's Creative Commons licence, unless indicated otherwise in a credit line to the material. If material is not included in the article's Creative Commons licence and your intended use is not permitted by statutory regulation or exceeds the permitted use, you will need to obtain permission directly from the copyright holder. To view a copy of this licence, visit http://creativecommons.org/licenses/by/4.0/.

\section{References}

1. Recht L, van den Bent M, Shih H (2020) Treatment and prognosis of diffuse (grade II) and anaplastic (grade III) astrocytomas in adults [Internet]. Post TW, ed. UpToDate. Waltham, MA: UpToDate Inc. Available via: https://www.uptodate.com/contents/treatment-andprognosis-of-diffuse-grade-ii-and-anaplastic-grade-iiiastrocytomas-in-adults. Accessed 31 Oct 2020

2. Straube C, Kessel KA, Schmidt-Graf F et al (2018) A trend towards a more intense adjuvant treatment of low-grade-gliomas in tertiary centers in Germany after RTOG 9802 - results from a multi-center survey. BMC Cancer 18:907

3. Sepúlveda-Sánchez JM, Muñoz Langa J, Arráez MÁ et al (2018) SEOM clinical guideline of diagnosis and management of lowgrade glioma (2017). Clin Transl Oncol 20:3-15

4. National Institute for Health and Care Excellence (2018) Brain tumours (primary) and brain metastases in adults. National Institute for Health and Care Excellence, London [Internet]. 2018. Available via: https://www.nice.org.uk/guidance/ng99. Accessed 31 Oct 2020

5. Wang J, Zheng X, Zhang J et al (2020) An MRI-based radiomics signature as a pretreatment noninvasive predictor of overall survival and chemotherapeutic benefits in lower-grade gliomas. Eur Radiol https://doi.org/10.1007/s00330-020-07581-3

6. Park CJ, Han K, Kim H et al (2020) Radiomics risk score may be a potential imaging biomarker for predicting survival in isocitrate dehydrogenase wild-type lower-grade gliomas. Eur Radiol 30: 6464-6474

7. Choi YS, Ahn SS, Chang JH et al (2020) Machine learning and radiomic phenotyping of lower grade gliomas: improving survival prediction. Eur Radiol 30:3834-3842

8. van Timmeren JE, Cester D, Tanadini-Lang S, Alkadhi H, Baessler B (2020) Radiomics in medical imaging - "how-to" guide and critical reflection. Insights Imaging 11:91

9. Pinto dos Santos D, Dietzel M, Baessler B (2020) A decade of radiomics research: are images really data or just patterns in the noise? Eur Radiol. https://doi.org/10.1007/s00330-020-07108-w

10. Baeßler B, Weiss K, Pinto dos Santos D (2019) Robustness and reproducibility of radiomics in magnetic resonance imaging: a phantom study. Invest Radiol 54:221-228

Publisher's note Springer Nature remains neutral with regard to jurisdictional claims in published maps and institutional affiliations. 